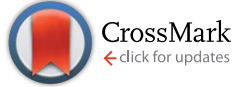

Cite this: Soft Matter, 2014, 10, 6457

Received 19th May 2014

Accepted 25th June 2014

DOI: $10.1039 / \mathrm{c} 4 \mathrm{sm} 01093 \mathrm{k}$

www.rsc.org/softmatter

\section{The adsorption-desorption behaviour and structure function relationships of bile salts}

\begin{abstract}
Roger Parker, ${ }^{\star}$ Neil M. Rigby, Michael J. Ridout, A. Patrick Gunning and Peter J. Wilde*
The digestion of dietary components in the human gastrointestinal (Gl) tract is a complex, dynamic, inherently heterogeneous process. A key aspect of the digestion of lipid in the Gl tract is the combined action of bile salts, lipase and colipase in hydrolysing and solubilising dispersed lipid. The bile salts are a mixture of steroid acid conjugates with surfactant properties. In order to examine whether the different bile salts have different interfacial properties their dynamic interfacial behaviour was characterised. Differences in the adsorption behaviour to solid hydrophobic surfaces of bile salt species were studied using dual polarisation interferometry and atomic force microscopy (AFM) under physiological conditions. Specifically, the cholates adsorbed more slowly and a significant proportion were irreversibly adsorbed following buffer rinsing; whereas the deoxycholates and chenodeoxycholates adsorbed more rapidly and desorbed to a greater extent following buffer rinsing. The conjugating groups (taurine, glycine) did not influence the behaviour. AFM showed that the interfacial structures that remained following buffer rinsing were also different between these two groups. In addition, the adsorptiondesorption behaviour affected the adsorption of colipase to a solid surface. This supports the idea that cooperative adsorption occurs between certain bile salts and colipase to facilitate the adsorption and activity of pancreatic lipase in order to restore lipolytic activity in the presence of bile salts. This study provides insights into how differences in bile salt structure could affect lipase activity and solubilisation of lipolysis products and other lipid-soluble bioactive molecules.
\end{abstract}

\section{Introduction}

In order to develop foods which may modulate the rate and location at which they deliver digestion products within the gastrointestinal tract it is necessary to have a deeper understanding of the digestion process. In particular, the small intestinal processing of lipids occurs through the mixing of the food with pancreatic secretions, bile from the liver and bicarbonate from the epithelium (e.g. from Brunner's glands). Lipase catalyses the hydrolysis of triacylglycerides to fatty acids, diglycerides and monoglycerides which form mixed micelles with other surface active species from which they can be absorbed into the intestinal epithelium. Bile salts, major components of bile, are surface active and play a key role at interfaces in combination with lipase and colipase. Their role is to displace existing surface active material, promote the adsorption of lipase and colipase, facilitate the removal of lipolysis product from the interface and form part of the mixed micelles which solubilise the lipolysis products. ${ }^{1-3}$

Bile typically contains $25-35 \% \mathrm{w} / \mathrm{w}$ bile salts. ${ }^{4}$ Bile salts are conjugates of bile acids (derived by cholesterol oxidation in the liver) with the amino acids glycine and taurine. Whereas the

Institute of Food Research, Norwich Research Park, Colney, Norwich, NR4 7UA, UK. E-mail: roger.parker@ifr.ac.uk; peter.wilde@ifr.ac.uk cholates (NaTC, NaGC) and the chenodeoxycholates (NaTCDC and NaGCDC) are synthesised in the liver and are termed primary bile salts, the deoxycholates (NaTDC and NaGDC) are formed by the dehydroxylation of the cholate largely in the colon. All the bile salts are susceptible to deconjugation to bile acids in the small intestine. Measurements have shown that 95\% of the bile acids are readsorbed before leaving the ileum (the lower part of the small intestine) from where they travel back to the liver via the hepatic portal vein and are retransformed to the original mix of conjugated bile salts ready for resecretion into the duodenum either directly from the liver or after storage in the gall bladder. This cycling is known as the "enterohepatic circulation". The bile salts have different abundances in bile and an average composition ${ }^{5}$ is given in Table 1. The glycoconjugates form about $70 \%$ of the mixture and the tauroconjugates $30 \%$. The cholates and chenodeoxycholates each comprise about $41-43 \%$, the deoxycholates comprise about $12 \%$ and minor bile acids make up the remaining $5 \%$.

The micellar properties of the bile salts were recently reviewed by Madenci and Egalhaaf, ${ }^{6}$ Table 1 . The glycoconjugates have well defined critical micelle concentrations in the range 1-4 $\mathrm{mM}$ and aggregation numbers ranging from 9-16. In contrast, the tauroconjugate cmc's are less well defined and occur at higher concentrations, particularly taurocholate (3-18 $\mathrm{mM}$ ) and, to a lesser extent, taurochenodeoxycholate 
Table 1 The main bile salt abundance in gall bladder bile from Rossi et $a .^{5}$ and critical micelle concentration $(\mathrm{cmc})$ and aggregation number $\left(N_{\text {agg }}\right)$ from Madenci and Egelhaaf ${ }^{6}$

\begin{tabular}{lllll}
\hline Bile salt (all sodium) & Abbreviation & Mol/\% & Cmc/mM & $N_{\text {agg }}$ \\
\hline Taurocholate & NaTC & 10.9 & $3-18$ & $3-7$ \\
Glycocholate & NaGC & 25.6 & 4 & 9 \\
Taurochenodeoxycholate & NaTCDC & 12.8 & $0.9-7$ & $5-26$ \\
Glycochenodeoxycholate & NaGCDC & 25.7 & $1-2$ & 15 \\
Taurodeoxycholate & NaTDC & 4.7 & $2-3$ & $12-19$ \\
Glycodeoxycholate & NaGDC & 11.1 & $1-2$ & $13-16$ \\
\hline
\end{tabular}

(0.9-7 $\mathrm{mM})$ and they have correspondingly smaller aggregation numbers.

In the small intestine triglycerides are hydrolysed by pancreatic lipase. However, the presence of bile salts, necessary to remove lipolysis products and polar lipids from the interface, also inhibit pancreatic lipase activity. ${ }^{7}$ Therefore, pancreatic lipase requires the cofactor colipase in order to act effectively in the presence of bile salts. ${ }^{7,8}$ Colipase has a molecular mass about $10 \mathrm{kDa}$ with 93 amino acids. Its structure is stabilised by five disulphide bonds. X-ray ${ }^{9,10}$ and $\mathrm{NMR}^{11}$ studies identified a four fingered structure of surface loops tipped with non-polar residues which lead to its adsorption at triglyceride-water interfaces. It also forms a $1: 1$ complex with lipase, tethering it to the triglyceride-aqueous phase interface and holding its "lid" domain open allowing the substrate access to its catalytic site. ${ }^{12}$

From an interface chemistry perspective, the process of pancreatic lipolysis is intriguing, particularly the action of the bile salts. Their chief function is the removal of material from the surface to enable lipase to access the interface, and to remove lipolysis product. In contrast, in the presence of colipase, they also promote the adsorption of the enzyme and its cofactor. It seems unlikely that a single surfactant could perform these two very different roles in a self-assembled system. However, the fact that there are several different forms of bile salt could help address this issue, allowing different bile salts to perform different roles during the duodenal lipid digestion process.

Therefore the aim of this study was to determine whether the structure of individual bile salts influences their interfacial behaviour and interaction with the lipase cofactor colipase. We determined the adsorption-desorption properties of individual bile salts and the lipase cofactor colipase at a model hydrophobic solid-liquid interface. Dual polarisation interferometry (DPI) with a C18-modified silicon oxide sensor was used. DPI is an optical technique which is very sensitive to adsorbed mass, and rapid solvent exchange allows adsorption-desorption kinetics to be quantified. Firstly, the behaviours of the individual pure bile salts were studied, to determine any structurefunction relationships, followed by a study of the various interactions of the bile salts with colipase in order to understand whether the structure of the different bile salts could be responsible for the multi-functionality of the bile. The microand nanoscale structural changes resulting from adsorption and desorption of bile salts from a molecularly-smooth hydrophobic surface were also imaged using atomic force microscopy (AFM).

\section{Material and methods}

\section{Materials}

Sodium taurocholate (NaTC), sodium glycocholate (NaGC), sodium taurodeoxycholate (NaTDC), sodium glycodeoxycholate (NaGDC), sodium taurochenodeoxycholate (NaTCDC) and sodium glycochenodeoxycholate (NaGCDC) were obtained from Sigma (see Fig. 1 for structures). Porcine colipase was from Roche. Bis-tris, sodium chloride, calcium chloride, ethanol, propanol and SDS were also from Sigma. The solutions were made up in $\mathrm{pH}$ 7.0, $2 \mathrm{mM}$ bis-tris buffer containing $150 \mathrm{mM}$ $\mathrm{NaCl}$ and 0,1 or $10 \mathrm{mM} \mathrm{CaCl}_{2}$. Water was from a SG Labostar water purification system (Siemens) fed with deionised water.

\section{Dual polarisation interferometry (DPI)}

A dual polarisation interferometer from Biolin (formerly Farfield Scientific, model Analight Bio200) determines the mass and thickness of a film adsorbed onto the surface of an optical sensor. The sensor is a waveguide, and the adsorbed films properties are interrogated by the evanescent wave resulting from reflections from the internal surface of the waveguide. Changes in the resulting interference pattern with a reference waveguide are used to calculate the adsorbed layer thickness, mass and adsorbed species concentration. Measurements are interpreted using a model which assumes the adsorbed molecules form a layer of uniform thickness and refractive index. The results have been processed assuming a refractive index increment, $\mathrm{d} n / \mathrm{d} c$, of $0.20 \mathrm{~mL} \mathrm{~g}^{-1}$. The calculation uses the average of the refractive indices of the solvent, the refractive index of the adsorbed layer and the refractive index increment to calculate the mass concentration $\left(\mathrm{mg} \mathrm{mL}^{-1}\right)$ in the adsorbed layer. With the thickness, this allows mass adsorbed per unit area to be calculated. Refractive index increments were determined using a refractive index detector (Shodex RI-71, Showa Denko) with solutes dissolved in the running buffer at concentrations between $0-2.5 \mathrm{mg} \mathrm{mL} \mathrm{m}^{-1}$. The precision of the measurement technique was checked using sucrose and $\mathrm{NaCl}$ solutions and was found to be within $3 \%$ of literature values.

A bile salt adsorption-desorption experiment was performed by first establishing a baseline response in the running buffer flowing at $15 \mu \mathrm{L} \mathrm{min}{ }^{-1}$, injecting the bile salt solution for $300 \mathrm{~s}$ at $15 \mu \mathrm{L} \mathrm{min}^{-1}$, followed by a running buffer rinse for $300 \mathrm{~s}$ at 15 $\mu \mathrm{L} \mathrm{min}^{-1}$. This was followed by a $40 \% \mathrm{w} / \mathrm{w}$ ethanol-water cleaning step $\left(120 \mathrm{~s}, 50 \mu \mathrm{L} \mathrm{min}{ }^{-1}\right)$ prior to the next experiment $240 \mathrm{~s}$ later.

Colipase-bile salt adsorption-desorption experiments comprised two stages, firstly, a series of injections of increasing concentrations of colipase (300 s at $15 \mu \mathrm{L} \mathrm{min}{ }^{-1}$ followed by 300

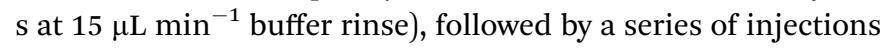
of bile salts at increasing concentrations.

Colipase-bile salt coadsorption-desorption experiment comprised three stages: (1) the colipase-bile salt mixture was injected at $15 \mu \mathrm{L} \mathrm{min}{ }^{-1}$ for $300 \mathrm{~s}$, then the flow was stopped and 


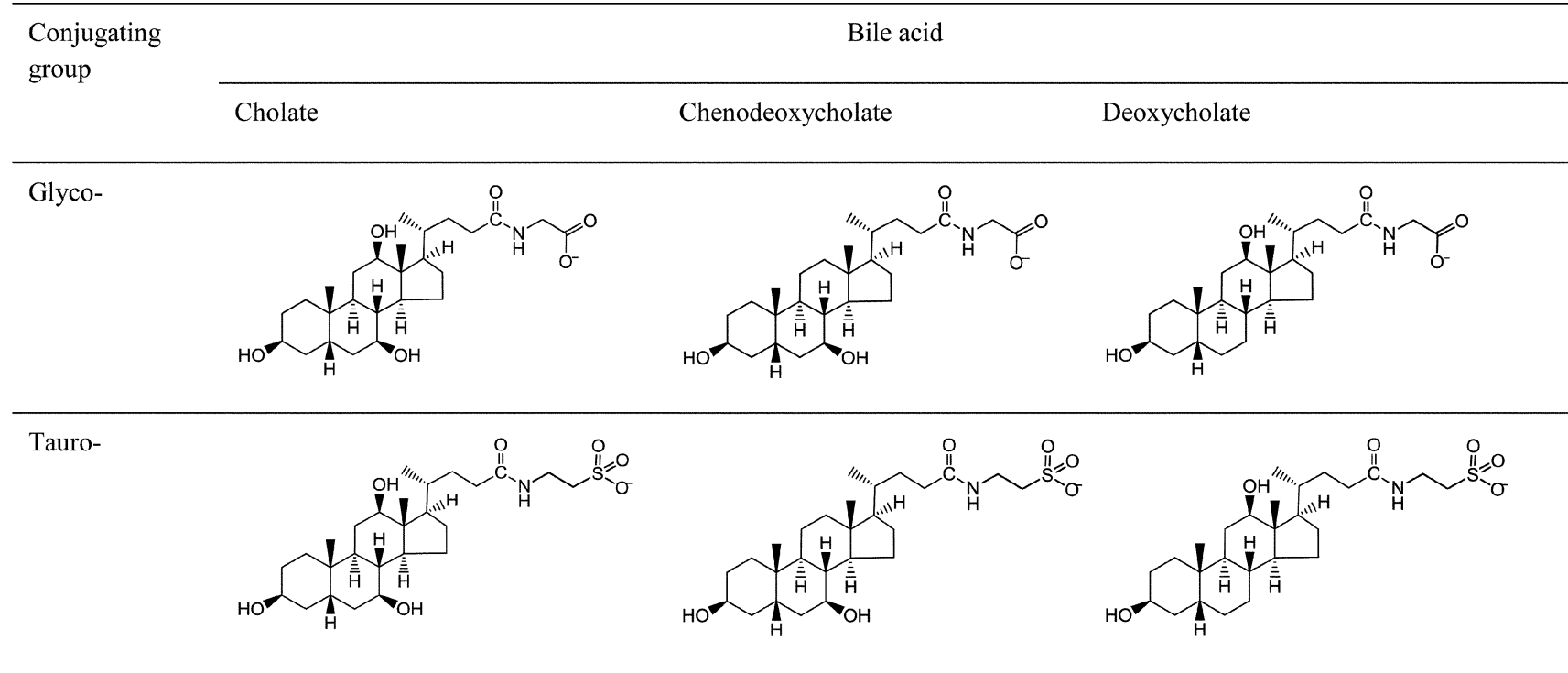

Fig. 1 Bile salt structures. The hydroxyls are at positions 3 (on left), 7 (bottom right) and 12 (uppermost) on the bile acid.

the injection valve closed for $60 \mathrm{~s}$ during which time the loop was filled with bile salt solution at the same concentration; (2) the injection valve was opened and the flow restarted at $15 \mu \mathrm{L}$ $\mathrm{min}^{-1}$ for $600 \mathrm{~s}$ to rinse the adsorbed layer with bile salt solution; (3) finally the injection valve was closed allowing a $600 \mathrm{~s}$ buffer rinse.

\section{Atomic force microscopy (AFM)}

The atomic force microscope used was an MFP-3D BIO (Asylum Research, Santa Barbara, CA, USA). Images were acquired in AC mode in air using AC160TS cantilevers (Olympus, Japan) oscillated $10 \%$ below their fundamental resonant frequency (typically around $320 \mathrm{kHz}$ ). The feedback loop set-point was typically around $8 \%$ damping of the free amplitude, to minimise the force exerted on the sample. The scan rate was $1 \mathrm{~Hz}$. The experiments were performed on highly oriented pyrolitic graphite (HOPG) to satisfy the twin requirements of a hydrophobic surface sufficiently flat to allow visualisation of the bile salts at monolayer resolution. Adsorption of the bile salts onto the HOPG and subsequent rinsing was performed in a sealed flow cell: the HOPG was incubated in bile salt solutions $(10 \mathrm{mM}$ in the bis-tris buffer described above) for 8 min at $21{ }^{\circ} \mathrm{C}$, and then the cell was rinsed with 7.5 volumes of buffer, followed by 6 volumes of ultrapure water $(18.2 \mathrm{M} \Omega \mathrm{cm})$. The final rinse was required to eliminate crystallisation of the buffer salts on the surface of the HOPG. The sample was removed from the cell and any excess water tipped off the HOPG surface and left for $30 \mathrm{~min}$ to fully dry before imaging.

\section{Results and discussion}

\section{Bile salt adsorption-desorption}

Fig. 2 shows duplicate runs of a adsorption-desorption experiment in which a C18 chip is exposed to $3 \mathrm{mM}$ NaTC (a) and NaGDC (b) solution in standard buffer for $300 \mathrm{~s}$ (flow rate
$15 \mu \mathrm{L} \min ^{-1}$ ) followed by running buffer rinsing for $300 \mathrm{~s}$ at the same flow rate before cleaning the chip for the next experiment.
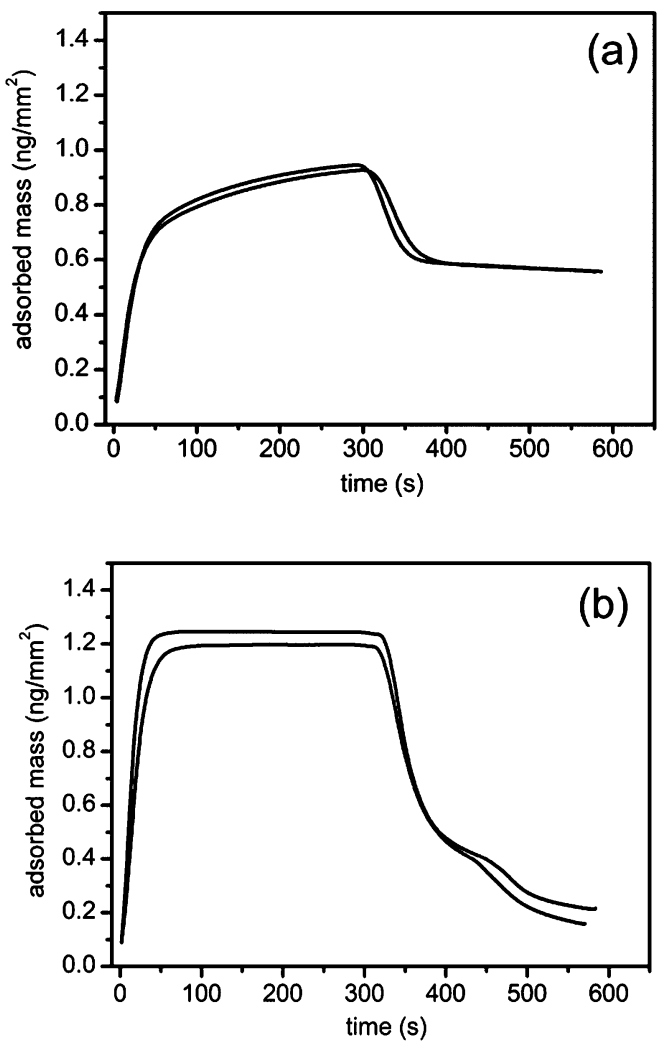

Fig. 2 Adsorption-desorption of bile salts on a C18 surface measured with a dual polarisation interferometer. Bile salts: (a) NaTC; (b) NaGDC; concentration, $3 \mathrm{mM}$; $\mathrm{pH}$, 7.0; buffer: $2 \mathrm{mM}$ bis-tris $150 \mathrm{mM} \mathrm{NaCl}$,

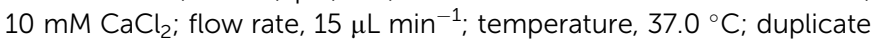
measurements. 
The adsorption of the two bile salts show contrasting behaviour. The measurement cell takes about $70 \mathrm{~s}$ to completely exchange with sample at this flow rate. The adsorption of the NaTC continues to increase after complete exchange indicating some slow process within the adsorbed layer which allows increasing adsorption. After the $300 \mathrm{~s}$ injection, during the rinsing phase, a proportion of the adsorbed layer desorbs within the cell exchange time but a large proportion of the material is retained on the chip surface. In contrast the NaGDC adsorption is complete within the cell exchange time and the adsorption peak is flat topped indicating that adsorption has reached steady state within the cell exchange time. In the rinsing phase over $80 \%$ of the adsorbed NaGDC is lost indicating the adsorption is largely reversible over this timescale.

The adsorption-desorption processes were investigated over a wider range of bile salt concentrations from $0.1 \mathrm{mM}$ to $10 \mathrm{mM}$. Fig. 3 shows the mass adsorbed after $300 \mathrm{~s}$ adsorption and after $300 \mathrm{~s}$ rinsing for (a) NaTC and (b) NaGDC. The onset of the NaTC adsorption irreversibility is at low concentration at about 0.2-0.3 $\mathrm{mM}$ and occurs up to the highest concentration studied. The more reversible adsorption of the NaGDC was observed throughout the concentration range with the exception of the 2 $\mathrm{mM}$ solution. This solution showed a higher level of adsorption,
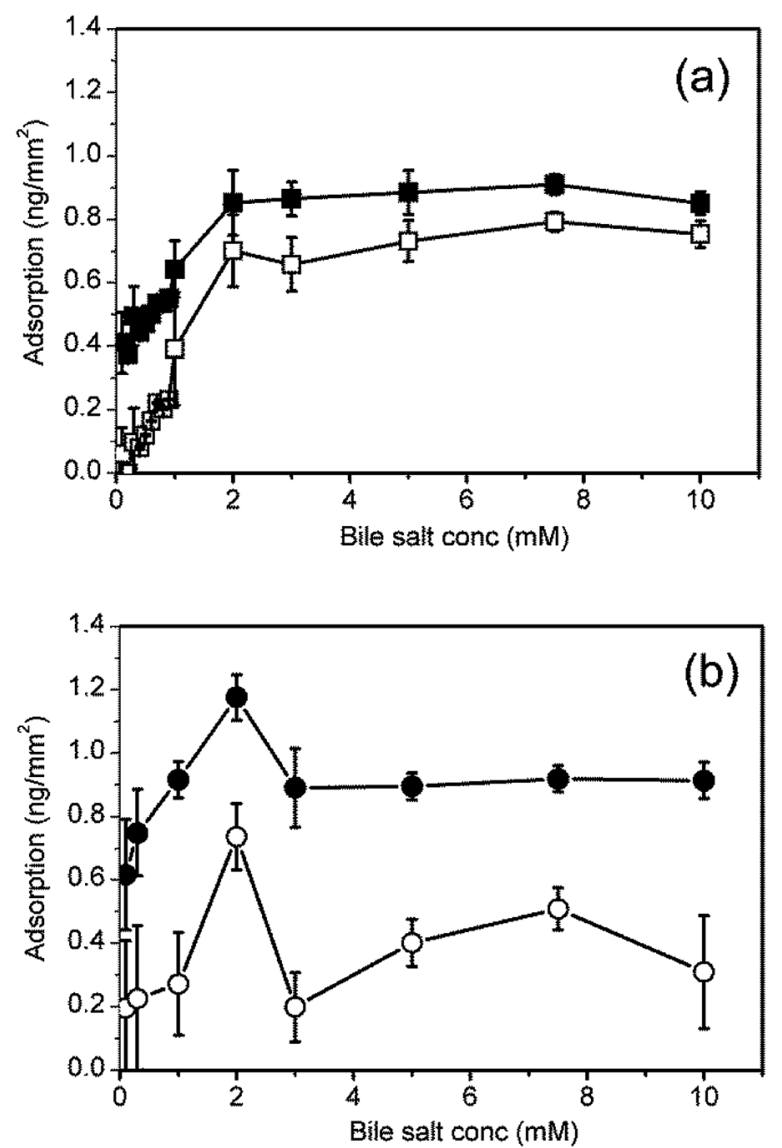

Fig. 3 Bile salt adsorption isotherms on a C18 surface. Bile salts: (a) $\mathrm{NaTC}$; (b) NaGDC. Filled symbols represent the amount adsorbed at end of the adsorption step; open symbols represent the amount adsorbed at the end of the rinsing step. a sustained increase in adsorption similar to that exhibited by NaTC in Fig. 2(a) and was not wholly removed by the standard $40 \% \mathrm{w} / \mathrm{w}$ ethanol-water rinse. However on increasing the NaGDC concentration to $3 \mathrm{mM}$, more reversible behaviour was again observed. The lack of complete desorption was an unexpected result, as soluble, micelle forming surfactants are normally expected to fully desorb from surfaces following a rinsing step. ${ }^{\text {13-15}}$ This is because, as the subphase is rinsed, the surfactant concentration drops below the cmc and the surfactant will begin to desorb to maintain equilibrium with the bulk solution until all the solute is removed and the surfactant completely desorbs. ${ }^{16}$ Only polymeric surfactants such as proteins will irreversibly adsorb to surfaces, with only limited desorption following rinsing. ${ }^{\mathbf{1 4 , 1 5 , 1 7}}$

Duan et al. recently reported the results of a study of the adsorption-desorption behaviour of the sodium dodecyl sulphate (SDS) using dual polarisation interferometry. ${ }^{18}$ The variation of mass adsorbed with surfactant concentration has the same qualitative features as NaGDC (Fig. 3(b)). In both cases the peak in adsorption is close to the critical micelle concentration. The Duan et al. study includes a detailed description of the form of the adsorption behaviour both above and below the cmc. For the compositions above the cmc there is a rapid adsorption phase followed by a plateau, as in Fig. 2(b). This indicates that the micellar structure in the bulk is having an influence on the adsorption process. Similarly, the initial rapid desorption of NaGDC (Fig. 2(b)) may be due to the transient shedding of micelles as the surface is rinsed with bile-free buffer. At bulk concentrations in a range below the cmc, SDS adsorbs on hydrophobic surfaces and forms hemimicelles. ${ }^{18}$ In this concentration range the SDS adsorption behaviour is similar to Fig. 2(a), NaTC, with a slow adsorption phase after the initial rapid increase, suggesting this may be a general type of adsorption behaviour for surfactants below their cmc.

The above studies have used a standard buffer: $2 \mathrm{mM} \mathrm{pH} 7.0$ bis-tris with $150 \mathrm{mM} \mathrm{NaCl}$ and $10 \mathrm{mM} \mathrm{CaCl}_{2}$ and two representative bile salts. In order to examine the effects of molecular structure and calcium level, all six bile salts (Table 1) were tested at three levels of calcium i.e. $0 \mathrm{mM}, 1 \mathrm{mM}$ and $10 \mathrm{mM}$. The

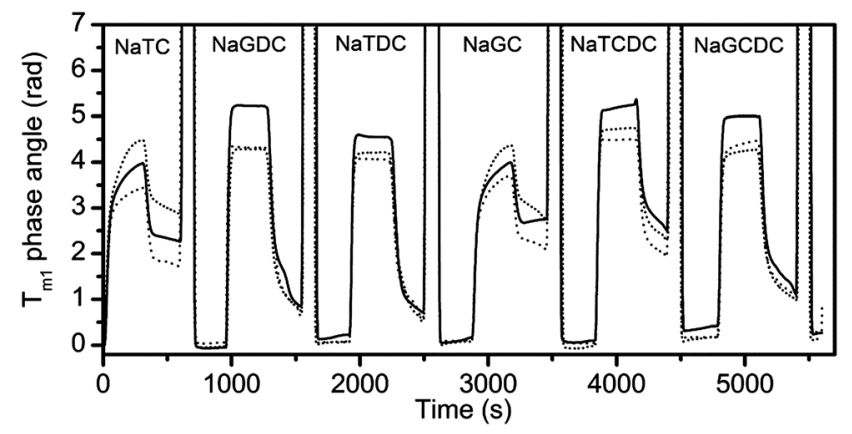

Fig. 4 Phase angle variation for transverse magnetic component of light during sequential injection of six different bile salts $(3 \mathrm{mM})$. Each bile salt adsorption experiment is terminated by a 2 min ethanol-water cleaning injection $(40 \% \mathrm{w} / \mathrm{w})$ (signal offscale). Standard buffer with varying calcium chloride concentration (solid line, $0 \mathrm{mM}$; dashed line, $1 \mathrm{mM}$; dotted line, $10 \mathrm{mM}$ ). 
variation of the phase angle measured with transverse magnetic polarized light on channel 1 of the sensor chip is shown in Fig. 4 with results of the three calcium concentrations superimposed for a bile salt concentration of $3 \mathrm{mM}$.

Fig. 4 shows that the mode of adsorption-desorption is dependent upon the bile acid (the unconjugated part of the bile salt) i.e. both the taurocholate and glycocholate show high retention on rinsing with running buffer. In contrast, both deoxycholates showed essentially reversible behaviour and the chenodeoxycholates showed intermediate behaviour with the taurine conjugate showing retention, while the glycine conjugate show reversibility. This is an interesting observation, since a major difference in structure, namely the conjugation with either taurine or glycine has little effect on adsorptiondesorption behaviour, whilst minor structural changes in the main part of the structure have a much bigger impact on behaviour. For example the difference between the cholate and deoxycholate forms is a single hydroxyl at position 7 (Fig. 1). Nevertheless, this small change tends to lead to a lower $\mathrm{cmc}$ and higher aggregation number in the deoxycholate forms (Table 1$)^{6}$ which could also influence the interaction with the interface and any surface aggregation which may occur.

It appears that, in these experiments, the essential surface active character of the bile salts is determined by the bile acid portion of the conjugate. As the bile acids are facial amphiphiles with the opposite faces of the planar steroidal fused ring structure being hydrophilic and hydrophobic, these intimatelylinked portions of the structure are able to dominate behaviour. The polar/ionic taurine and glycine conjugating groups are attached through a flexible four carbon chain meaning they are separated from the main amphiphilic group. At pH 7.0 and 150 $\mathrm{mM} \mathrm{NaCl}$ we anticipate glycine will be fully charged, as will the strongly acidic taurine, though ionic interactions will be screened by the salt. These conjugating groups serve to increase the aqueous solubility of the bile salts but do so in a similar way, so maintaining the dominant role of the bile acid group.

The rates of adsorption and desorption are quantified the midpoint of the injection and the rinse for the adsorptiondesorption experiment with the bile salts containing $10 \mathrm{mM}$ $\mathrm{CaCl}_{2}$ in Table 2. This clearly demonstrates the range of behaviour. At this time point the cholates have finite on-rates and conversely zero off-rates, whereas the deoxycholates have zero on-rates and finite off-rates. The chenodeoxycholates are most similar to the deoxycholates though they have finite but

Table 2 Bile salt on- and off-rates at midpoint of injection (150 s) and rinse (450 s). Standard conditions and $10 \mathrm{mM} \mathrm{CaCl}_{2}$

\begin{tabular}{lcc}
\hline & $\mathrm{d} m / \mathrm{d} t\left(10^{-6} \mathrm{ng} \mathrm{mm}^{-2} \mathrm{~s}^{-1}\right)$ & \\
\cline { 2 - 3 } Bile salt & On-rate & Off-rate \\
\hline NaTC & $1160 \pm 150$ & $-250 \pm 210$ \\
NaGC & $1140 \pm 140$ & $-150 \pm 220$ \\
NaTCDC & $100 \pm 90$ & $-3000 \pm 2000$ \\
NaGCDC & $240 \pm 40$ & $-1600 \pm 420$ \\
NaTDC & $-80 \pm 70$ & $-2600 \pm 600$ \\
NaGDC & $-20 \pm 100$ & $-2200 \pm 340$
\end{tabular}

small on-rates. In summary there is a correlation between the sustained adsorption (finite on-rates) and slow desorption (zero off-rates).

Quantitative data showing the effects of calcium on bile salt adsorption and retention after buffer rinsing is shown in Fig. 5(a) and (b), respectively. Initial addition of calcium to 1 $\mathrm{mM}$ results in a $25-40 \%$ increase in bile salt adsorption. A further increase in calcium to $10 \mathrm{mM}$ results in a decrease in adsorption for the two cholates resulting in a net increase of about $10 \%$ as compared with the calcium free buffer. The adsorption of the other bile salts is little altered at the highest calcium concentration.

The effects of calcium level on the levels of adsorption after rinsing are relatively small. The initial addition of calcium results in a $25-30 \%$ reduction in the amount adsorbed for NaGCDC, NaTDC and NaGDC. At the highest concentration of $10 \mathrm{mM}$ calcium the largest net effect is for NaTC and NaGC which are about $15 \%$ lower than their calcium free values.

Therefore, for the bile salts alone, there is a fundamental difference in the adsorption-desorption behaviour of the different bile salts, controlled mainly by the structure of the unconjugated part of the molecule. Even for the bile salts that appear to desorb more readily (i.e. NaTDC, NaGDC, NaGCDC), there is still a significant amount of adsorbed material
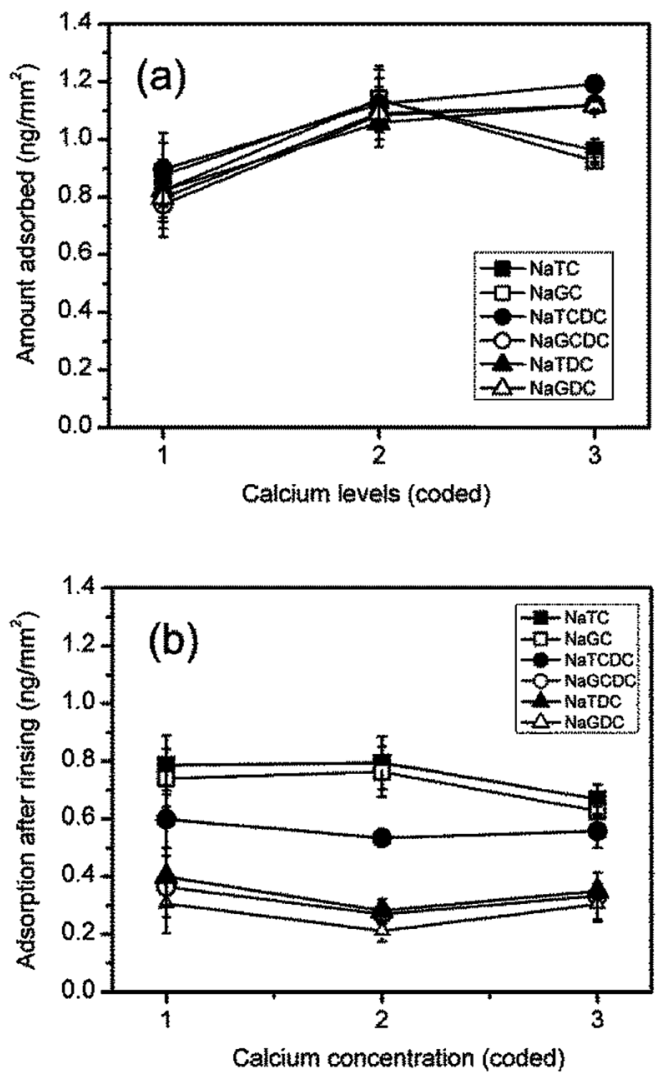

Fig. 5 Effect of calcium concentration of bile salt adsorption on a C18 surface. (a) Adsorption at end of adsorption step (300 s); (b) adsorption at end of buffer rinsing step. Calcium concentrations (coding): 1, $0 \mathrm{mM} ; 2,1 \mathrm{mM} ; 3,10 \mathrm{mM}$. Bile salts: $\mathbf{\square}, \mathrm{NaTC} ; \square, \mathrm{NaGC} ; \boldsymbol{\bullet}, \mathrm{NaTCDC}$; O, NaGCDC; $\mathbf{N}, \mathrm{NaTDC} ; \triangle, \mathrm{NaGDC}$. 
remaining after rinsing compared to studies with more conventional surfactants. ${ }^{\mathbf{1 3 , 1 4}}$ This suggests that the overall structure of the bile salts is affecting the rate and extent of desorption. Bile salts differ in their structure to conventional hydrocarbon-based surfactants which have a hydrocarbon, aliphatic chain and a polar headgroup, whereas bile salt molecules possess a planar steroidal fused-ring structure with hydrophobic and hydrophilic faces. This means that the adsorption, orientation and molecular interactions at interfaces will be completely different between the two classes of surface active molecule.

In order to try and elucidate the mechanism underpinning the adsorption-desorption behaviour of the bile salts, AFM imaging was used. Imaging the C18 modified silica surfaces used for the DPI measurements showed they were relatively rough (rms roughness approximately $9 \mathrm{~nm}$ ) at the molecular length scale which would make it difficult to resolve adsorbed bile salts on its surface (Fig. 6(a)). Highly oriented pyrolytic
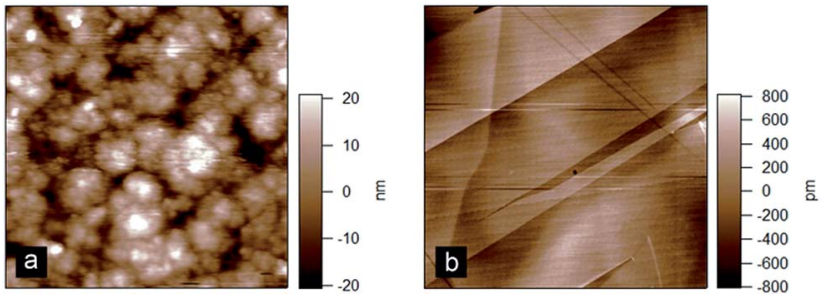

Fig. 6 Atomic force microscopy images of (a) C18 modified silicon oxide DPI chip and (b) highly oriented pyrolytic graphite. Scan size $=3 \mu \mathrm{m}$. graphite (Fig. 6(b)) offers a hydrophobic and atomically flat surface with an rms roughness of approximately $0.25 \mathrm{~nm}$ as compared with the bile salts which have approximate longest dimension $2 \mathrm{~nm}$ and thickness $0.5 \mathrm{~nm} .{ }^{19}$ Comparing the images, note that the graphite colour scale ranges from -800 to 800 pM (Fig. 6(b)) whereas that on the DPI chip (Fig. 6(a)) ranges from -20 to $20 \mathrm{~nm}$, which is a factor of 25 greater. Furthermore the surface of the DPI chip varies continuously in the $x$ and $y$ directions whereas the graphite has areas which are relatively smooth separated by clearly defined steps.

AFM images of NaTC and NaGDC adsorbed to a graphite surface were obtained after buffer rinsing. Fig. 7 shows some typical images obtained from numerous scans from several separate adsorption experiments. Fig. 7(a) and (b) show NaTC and NaGDC respectively, following adsorption and rinsing. Occasional holes were found in the film (black areas) which allowed measurement of the film thickness. NaTC films were approximately $2 \mathrm{~nm}$ thick, and formed a continuous film with a granular texture (granule diameter about $0.5 \mathrm{~nm}$ ), between any holes or defects. The granular structure is also evident around the edge of the hole. In contrast, NaGDC formed a more coarsely structured film, forming small "sheets" of about $100 \mathrm{~nm}$ across and $0.8 \mathrm{~nm}$ thick. The sheets were arranged in layers, of mainly one or two sheets, but sometimes three sheets in thickness. Certainly, the NaGDC adsorbed appeared to have been more disrupted by the rinsing/desorption step. These observations have to be taken in context, as the adsorption was onto a very different surface than the DPI measurements. Nevertheless, significant differences in the morphology of the adsorbed films were observed.
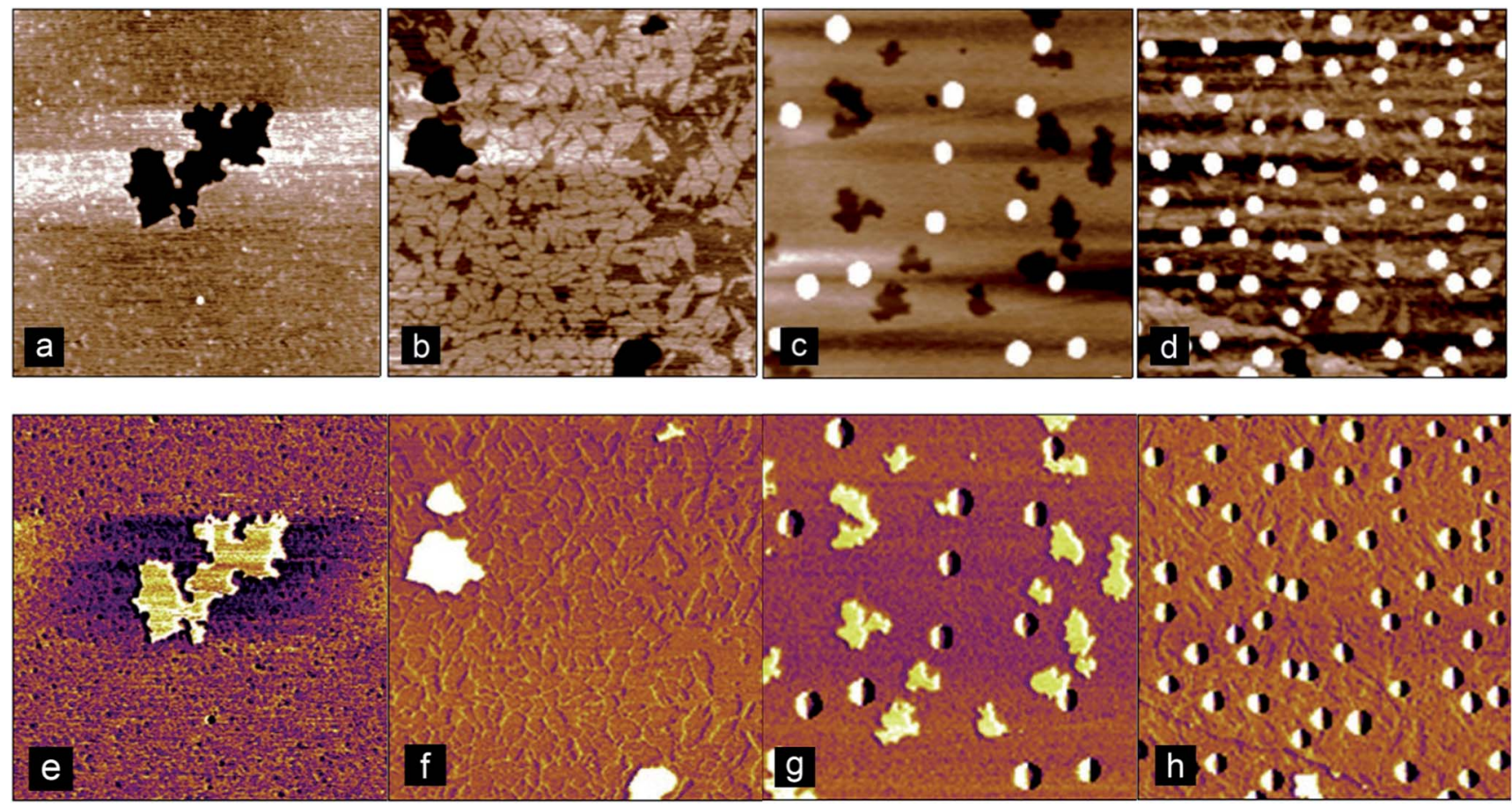

Fig. 7 Atomic force microscopy images of bile salts adsorbed onto HOPG after rinsing in buffer. (a and b) show NaTC and NaGDC, respectively. Black regions are holes in the adsorbed layer. (c and d) show NaTC and NaGDC respectively, in a region where micelle-like structures were observed. Scan size $=1.2 \mu \mathrm{m}$ in all images. Height ranges: (a) $1.2 \mathrm{~nm}$ : (b) $1.6 \mathrm{~nm}$; (c) $4.0 \mathrm{~nm}$; (d) $1.5 \mathrm{~nm}$. (e-h) are the corresponding phase images. 
In addition, on certain regions of the substrate, micelle-like structures were observed for both the NaTC (Fig. 7(c)) and NaGDC (Fig. 7(d)). These structures were of similar sizes between the two bile salts and were between 6 and $15 \mathrm{~nm}$ in height. There didn't appear to be any significant difference between the two bile salts in regard to the number and appearance of these structures, but it demonstrates that such supramolecular structures occur in these adsorbed-rinsed films.

\section{Bile salt-colipase adsorption-desorption on a C18 chip}

In the human small intestine bile salts interact with pancreatic lipase and colipase to digest triglyceride emulsions. Here these bile-protein interactions are examined using the cofactor colipase, which has long been known to bind to bile salts. ${ }^{7}$ Before describing coadsorption experiments, the results of a sequential adsorption experiment in which increasing amounts of colipase are first adsorbed onto the $\mathrm{C} 18$ chip followed by rinsing with increasing concentrations of bile salts are described.

Fig. 8(a) shows the raw data from the DPI for the transverse magnetic signal. In the first half of the experiment the C18 chip is exposed to increasing concentrations of colipase. Comparing the shape of the adsorption peaks between Fig. 8(a) (colipase) and Fig. 4 (bile salts) shows that, at the lowest colipase concentration $\left(0.01 \mathrm{mg} \mathrm{mL} \mathrm{m}^{-1}\right)$ the adsorption process is relatively slow and, from the buffer rinsing, largely irreversible. At higher colipase concentrations $\left(0.1-5 \mathrm{mg} \mathrm{mL}^{-1}\right)$ the signal increase slows after the first $60 \mathrm{~s}$ (about the cell exchange time) indicating that adsorption is relatively rapid and close to steady state. Fig. 8(b) shows the adsorption of colipase onto the C18 functionalised chip in the absence of any bile salts. The adsorption at the end of the adsorption phase is plotted prior to buffer rinsing. At the highest concentration studied $(5 \mathrm{mg}$ $\mathrm{mL}^{-1}$ ) the adsorption is approaching a constant value of about $1.9 \mathrm{ng} \mathrm{mm}^{-2}$, the mean optical thickness is $2.6 \mathrm{~nm}$ and the concentration is $0.74 \mathrm{~g} \mathrm{~mL}^{-1}$. The adsorption increases strongly between 1 and $10 \mu \mathrm{g} \mathrm{mL}{ }^{-1}$ reaching $76 \%$ of its final value at 10 $\mu \mathrm{g} \mathrm{mL}{ }^{-1}$.

In the second half of the experiment (Fig. 8(a)) the adsorbed colipase layer is rinsed with increasing concentrations of bile salts. The profile of the adsorption peak changes at $0.3 \mathrm{mM}$ NaGDC indicative of a desorption process occurring during the
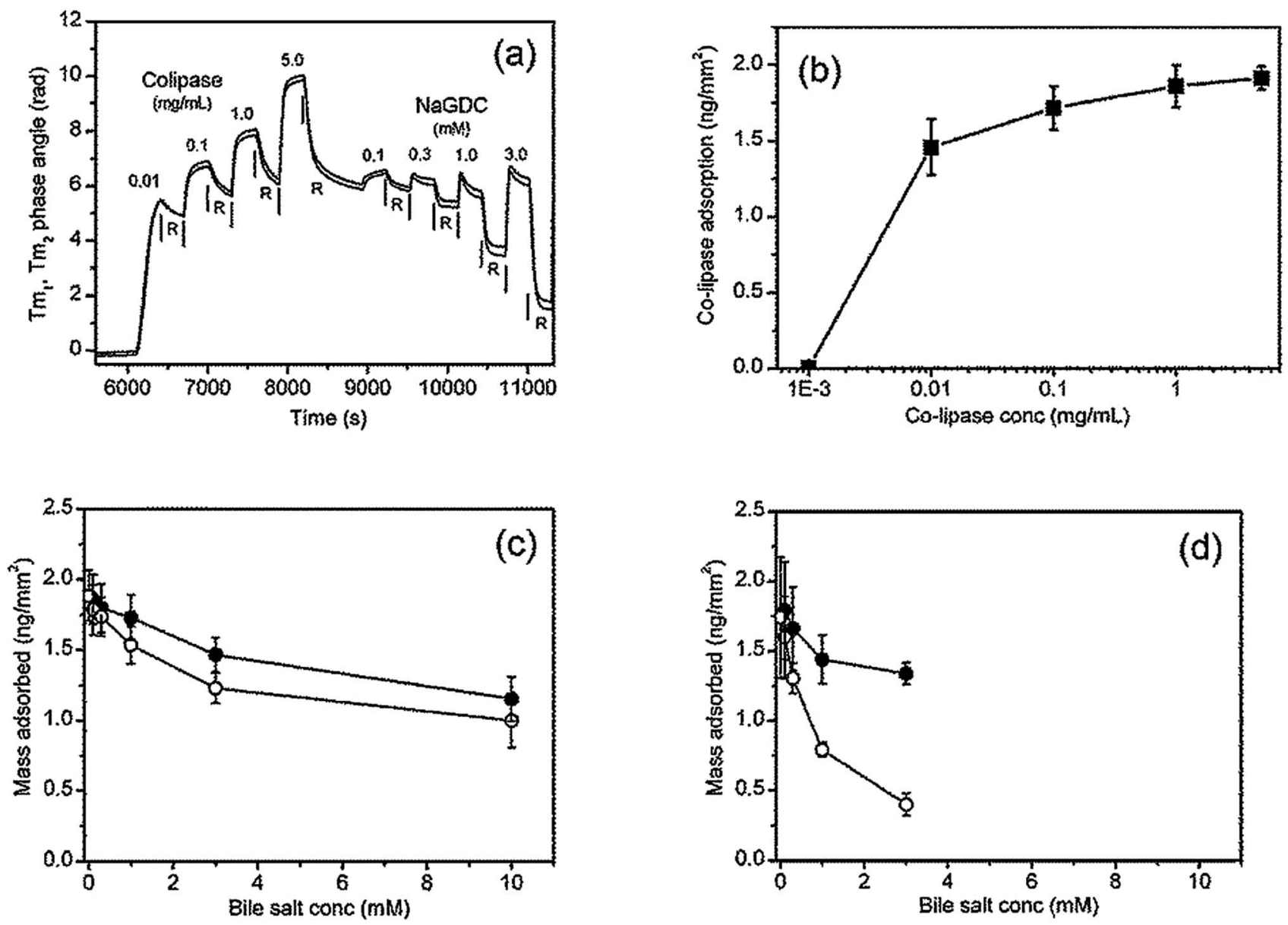

Fig. 8 Colipase adsorption and subsequent bile salt rinsing on C18 DPI chip. Raw data (a). The colipase is dissolved in $2 \mathrm{mM} \mathrm{pH} 7.0$ bis-tris buffer containing $150 \mathrm{mM} \mathrm{NaCl}$ and $10 \mathrm{mM} \mathrm{CaCl}_{2}$. The bile salt rinsing is achieved with a series of 300 s injections of increasing concentration each

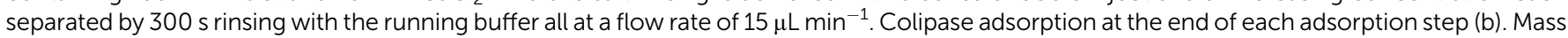
adsorbed on rinsing with bile salts: (c) NaTC; (d) NaGDC. Filled symbol denote the mass at end of bile rinsing step and open symbol the mass at the end of the buffer rinsing step. 
adsorption phase once the cell is fully exchanged. This effect is more pronounced in the $1.0 \mathrm{mM}$ and $3.0 \mathrm{mM}$ NaGDC injections. However, the largest reductions in adsorbed mass occurs during the buffer rinsing i.e. the rinsing of the mixed colipaseNaGDC layer with running buffer results in extensive desorption from the chip surface. The results of the rinsing experiments are summarised in Fig. 8(c) for NaTC and (d) for NaGDC. The bile salt rinsing of the adsorbed colipase layer reflects the differences in the bile salts observed in Fig. 2-5. The amounts adsorbed after buffer rinsing can be compared quantitatively at $3 \mathrm{mM}$. In the case of the pure bile salts (Fig. 3(a) and (b)) the amounts adsorbed after buffer rinsing (3 mM bile, $10 \mathrm{mM}$ $\mathrm{CaCl}_{2}$ ) are 0.66 and $0.29 \mathrm{ng} \mathrm{mm}^{-2}$ for NaTC and NaGDC, respectively, whereas for the mixed colipase-bile layers the amounts adsorbed after buffer rinsing are 1.23 and $0.40 \mathrm{ng}$ $\mathrm{mm}^{-2}$, respectively. That is, with NaTC, the mixed layers retain about $0.6 \mathrm{ng} \mathrm{mm}^{-2}$ extra material after buffer rinsing as compared with NaGDC which only retains $0.1 \mathrm{ng} \mathrm{mm}^{-2}$ extra material. Therefore, NaGDC is more efficient than NaTC at solubilising colipase from the surface when used in combination with buffer rinsing.

However, in order to more closely reflect the processes in the human small intestine, it is necessary to study the coadsorption of colipase and bile salts and the effect of rinsing with bile salts
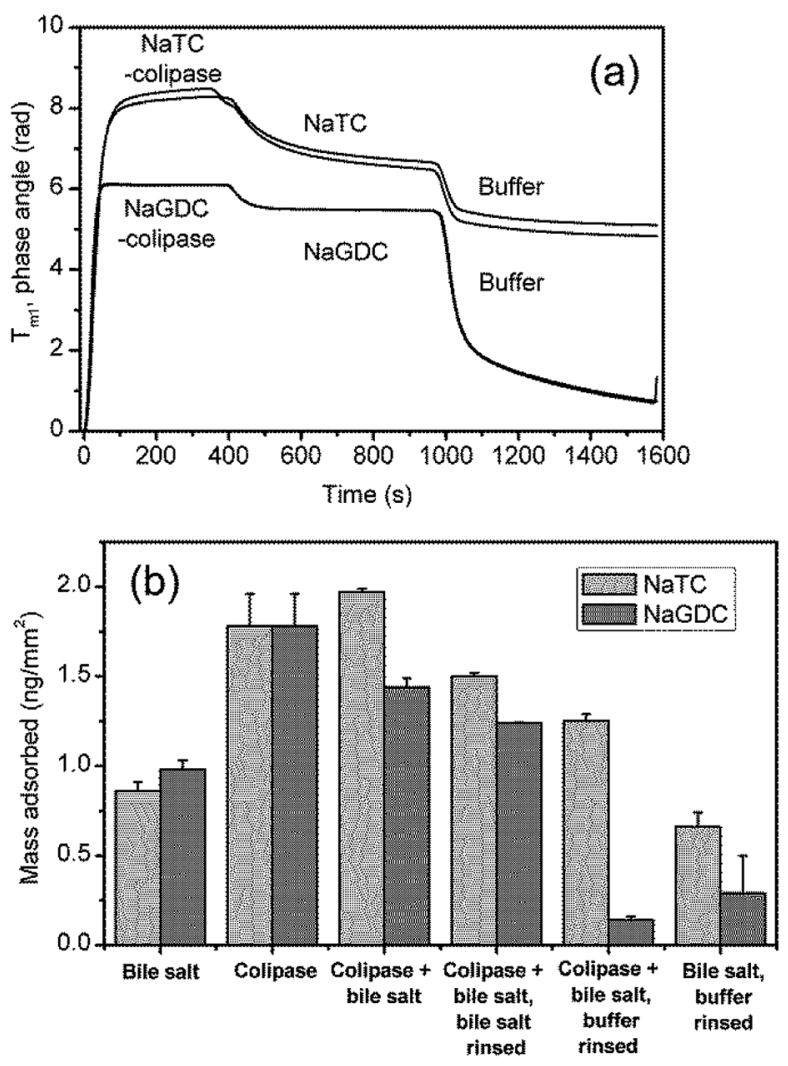

Fig. 9 Bile salt-colipase coadsorption, bile salt rinsing and buffer rinsing on $\mathrm{C} 18$ functionalised chip measured with DPI. Bile salt concentration, $3 \mathrm{mM}$; co-lipase concentration, $0.1 \mathrm{mg} \mathrm{mL}^{-1}$; buffer 2 $\mathrm{mM} \mathrm{pH} 7.0$ bis-tris with $150 \mathrm{mM} \mathrm{NaCl}$ and $10 \mathrm{mM} \mathrm{CaCl}_{2}$. (B). Results summary including bile adsorption (Fig. 3) and colipase adsorption (Fig. 6(a)). rather than running buffer. Results from experiments to investigate this are shown in Fig. 9. Fig. 9(a) and (b) show the combined adsorption of colipase and bile salt is greater for NaTC than for NaGDC and, while there are greater losses in adsorption for the NaTC on rinsing with the bile salt solution, a difference of $20 \%$ is maintained. Fig. 9(b) summarises the quantitative adsorption data including separate bile salt and colipase adsorption for comparison. The coadsorption experiment shows that the colipase and bile salts coadsorb but the extent of adsorption, while greater than just bile salt, is similar to the amount of adsorption for colipase. However, the coadsorption with NaTC is significantly greater than the coadsorption with NaGDC.

\section{Discussion}

Studies of the self-association properties of bile salts have shown that they have a range of behaviours in terms of critical micelle concentrations and aggregation numbers (Table 1). The present study shows that the individual bile salts have contrasting adsorption-desorption behaviour on C18-functionalised hydrophobic surfaces. Broadly, the behaviour of the bile salts split into two groups, those which have largely reversible adsorption behaviour (NaGDC, NaTDC and NaGCDC) and those which show a significant degree of irreversibility (NaTC, NaGC and NaGCDC). This grouping correlates with the self-association behaviour i.e. the reversibly adsorbed NaGDC, NaTDC and NaGCDC have the lower cmc's and larger aggregation numbers whereas the irreversibly adsorbed NaTC, NaGC and NaGCDC have higher cmc's and tend to aggregate into smaller micelles. This behaviour is finely tuned by the structure of the component bile acid, as they only differ by a single hydroxyl groups (Fig. 1) on $\mathrm{C} 12$ for the chenodeoxycholate and on C7 for the deoxycholate. Furthermore, this behaviour is insensitive to calcium levels which vary with dietary intake (Fig. 5). Although we refer to the adsorption behaviours as reversible or irreversible, as pointed out earlier, compared to freely soluble surfactants, even the reversible bile salts still have a proportion of the adsorbed fraction which remained adsorbed after rinsing. This was particularly noticeable at around $2 \mathrm{mM}$ (Fig. 3(b)). However, the duration of these experiments is quite short (though physiologically relevant), and it can be clearly seen from Fig. 5, that the desorption of NaGDC, NaTDC and NaGCDC is still continuing and persists to long times (3000 s, data not shown). Nevertheless, there is clearly a difference in the adsorption-desorption behaviour between the two sets of bile salts, with the bile salts which are slower to adsorb having a greater proportion of irreversibly adsorbed material. The link between bulk phase micellisation and interfacial adsorption-desorption suggests that bile salt "micelles" may be involved in the interfacial process. It was hoped that imaging the structure of the interfaces occupied by the bile salts may reveal whether they were in the form of aggregates at the interface. Fig. 6 showed that although the amount of adsorbed material appeared to be similar between the two bile salts, there were clear differences between the two in terms of the morphology. NaTC appeared to form an "amorphous" structure at the surface, with occasional 
holes, and evidence of adsorbed micelle-like structures. NaGDC, though, seemed to form more ordered, layered structures. It was not possible to image the bile salts on the DPI chip surface, as it was too rough. Although the HOPG graphite is hydrophobic, the surface chemistry is quite different. Nevertheless, the images shown in Fig. 6 clearly show that there was a difference in the adsorbed layer morphology between the two bile salts studied, which might help explain the differences in the adsorption-desorption behaviour.

In order to start to investigate whether these contrasting behaviours relate to physiological situations, the interaction of these bile salts with colipase was also investigated. The reasoning behind this was based on the fact that bile salts are necessary to remove insoluble lipolysis products (monoacylglycerol, free fatty acid) from the interface. This is because the accumulation of lipolysis product at the interface inhibits lipase activity, as the enzyme can no longer access the substrate. ${ }^{3,20}$ Removal of the product thus allows lipase access to the interface; however, the surface activity of the bile salts also inhibits the lipase. Therefore the cofactor colipase is present to facilitate the adsorption of lipase, and hence its activity, in the presence of bile salts. Colipase appears to interact with each of the other components: interface, lipase and bile. It is thought that it interacts with bile salts to aid adsorption of colipase to the interface. In turn, it binds strongly to lipase through its Cterminal which results in the complex having a sufficiently large hydrophobic footprint to remain adsorbed at the interface even in the presence of bile salts. ${ }^{\mathbf{8 , 2 1}}$ Furthermore, colipase binding also appears to stabilise the lipase "lid" conformation in the open position, ${ }^{\mathbf{8} 21}$ exposing the active site.

The present experiments show the interactions of three of the species present i.e. interface, bile salts and colipase. Fig. 9(b) summarises the adsorption data of species when they are adsorbed singly or simultaneously. The species and their interactions are shown schematically in Fig. 10. A comparison of the simultaneous adsorption of colipase and a bile salt with the adsorption of a bile salt alone, confirms that colipase can adsorb extensively in the presence of bile salts, thus supporting the idea of a cooperative adsorption process. However, the cooperativity appears to be greater for NaTC, than for NaGDC. Furthermore, the results for the effect of buffer rinsing of the coadsorbed layer show that the colipase adsorption is largely irreversible in the presence of NaTC which is consistent with its role during lipolysis. Yet in the presence of NaGDC, there has been significant desorption of both bile salt and colipase. These results support the premise that the differing adsorptiondesorption behaviours of the bile salt species could positively influence different phases of the lipolysis process. Specifically, NaTC-like behaviour would promote the adsorption and residence time of the colipase-lipase complex, and bile salts behaving like NaGDC would favour the displacement and solubilisation of lipolytic products from the interfacial region into the micellar phase.

The C18 functionalised surfaces represent a simplified model of a fat droplet surface with a layer of tethered 18 carbon alkyl chains. While the model provides hydrophobic chains to interact with the bile salts, other details of a fat droplet (a) $\mathrm{NaTC}$

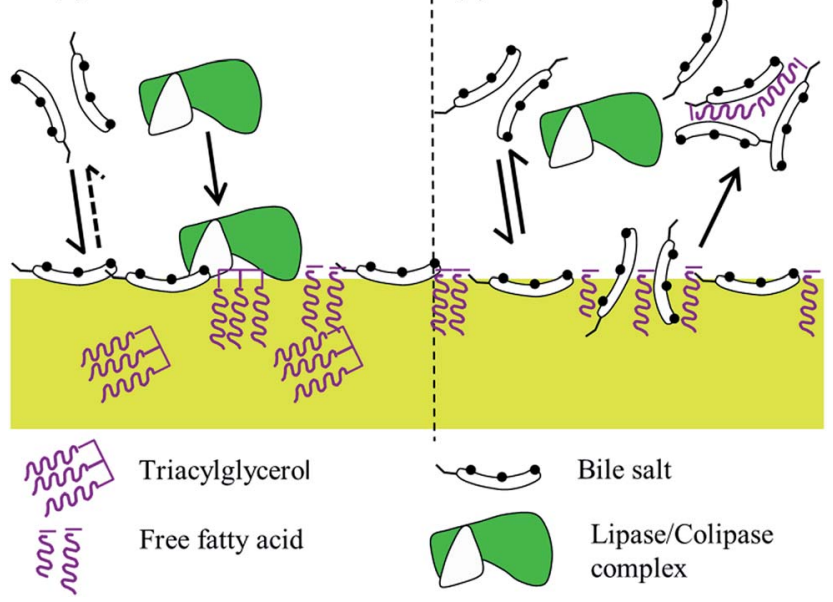

Fig. 10 Diagrammatic representation of the proposed action of bile salts. (a) NaTC adsorbs strongly, but only partially desorbs, leading to a longer residence time at the interface which facilitates the adsorption of lipase/colipase to the lipid surface, thus promoting lipolysis. In contrast, NaGDC (b) exchanges readily with the interface, which reduces the residence time at the interface, this may decrease adsorption but increase turnover of lipase/colipase. This behaviour may also facilitate the displacement of lipolytic products and their incorporation into mixed micelles.

undergoing lipolysis are absent e.g. the glycerol and carboxylic acid head groups of the triacylglycerols, monoacylglycerols and fatty acids. These species add to the complexity of the system both in terms of the molecular interactions (polar and charge) and in terms of the insoluble phases which they form. The approaches used in the present study rely on techniques employing solid-liquid interfaces which complement studies on the more complex hydrolysing liquid-liquid emulsion systems.

The results of the buffer rinsing of the coadsorbed layer are most relevant to conditions lower down the gastrointestinal tract. As part of the enterohepatic circulation almost all the bile salts are absorbed from the intestinal lumen and so their concentration will fall to low levels. Under these conditions while NaTC will tend to remain adsorbed to hydrophobic interfaces, the NaGDC will be released. However it is known that the colonic flora both deconjugate and dehydroxylate the bile salts, that is, they are biotransformed into other species low in the gastrointestinal tract. Therefore the ability of the bile salt species to remain adsorbed to surfaces may affect the bile salt population exposed to the colonic microflora.

\section{Conclusions}

Differences in the adsorption behaviour to solid surfaces between different bile salt species were observed. Specifically, the cholates adsorbed more slowly and a significant proportion were irreversibly adsorbed following buffer rinsing; whereas the deoxycholates and chenodeoxycholates adsorbed more rapidly and desorbed to a greater extent following buffer rinsing. AFM showed that the interfacial structures that remained following 
buffer rinsing were also different between these two groups. In addition, the adsorption desorption behaviour affected the adsorption of colipase to a solid surface. This supports the idea that cooperative adsorption occurs between certain bile salts and colipase to facilitate the adsorption and activity of pancreatic lipase in order to restore lipolytic activity in the presence of bile salts. Further work is required to understand how these subtle differences in bile salt structure affect lipase activity and solubilisation of lipolysis products and other lipidsoluble bioactive molecules.

\section{Acknowledgements}

The authors would like to thank the BBSRC for their support through Institute Strategic Programme Grant-Food and Health at the Institute of Food Research, Norwich.

\section{Notes and references}

1 H. I. Friedman and B. Nylund, Am. J. Clin. Nutr., 1980, 33, 1108-1139.

2 P. Reis, H. Watzke, M. Leser, K. Holmberg and R. Miller, Biophys. Chem., 2010, 147, 93-103.

3 P. J. Wilde, in Encyclopedia of Human Biology, Elsevier, 3rd edn, 2015, pp. 1-7.

4 Geigy Scientific Tables, ed. C. Lentner, Ciba-Geigy, 8th edn, 1981.

5 S. S. Rossi, J. L. Converse and A. F. Hofmann, J. Lipid Res., 1987, 28, 589-595.

6 D. Madenci and S. U. Egelhaaf, Curr. Opin. Colloid Interface Sci., 2010, 15, 109-115.
7 B. Borgstrom, C. Erlanson-Albertsson and T. Wieloch, J. Lipid Res., 1979, 20, 805-816.

8 M. E. Lowe, Annu. Rev. Nutr., 1997, 17, 141-158.

9 M. P. Egloff, F. Marguet, G. Buono, R. Verger, C. Cambillau and H. Vantilbeurgh, Biochemistry, 1995, 34, 2751-2762.

10 M. P. Egloff, L. Sarda, R. Verger, C. Cambillau and H. Vantilbeurgh, Protein Sci., 1995, 4, 44-57.

11 J. N. Breg, L. Sarda, P. J. Cozzone, N. Rugani, R. Boelens and R. Kaptein, Eur. J. Biochem., 1995, 227, 663-672.

12 D. C. Whitcomb and M. E. Lowe, Dig. Dis. Sci., 2007, 52, 1-17.

13 D. A. Woods, J. Petkov and C. D. Bain, J. Phys. Chem. B, 2011, 115, 7353-7363.

14 V. B. Fainerman, M. E. Leser, M. Michel, E. H. LucassenReynders and R. Miller, J. Phys. Chem. B, 2005, 109, 96729677.

15 T. F. Svitova, M. J. Wetherbee and C. J. Radke, J. Colloid Interface Sci., 2003, 261, 170-179.

16 J. Brinck, B. Jonsson and F. Tiberg, Langmuir, 1998, 14, 58635876.

17 A. Ash, M. J. Ridout, R. Parker, A. R. Mackie, G. R. Burnett and P. J. Wilde, Colloids Surf., B, 2013, 102, 546-553.

18 M. Duan, H. Wang, S. Fang and Y. Liang, J. Colloid Interface Sci., 2014, 417, 285-292.

19 A. R. Campanelli, S. C. Desanctis, A. A. Darchivio, E. Giglio and L. Scaramuzza, J. Inclusion Phenom., 1991, 11, 247-256.

20 P. J. Wilde and B. S. Chu, Adv. Colloid Interface Sci., 2011, 165, 14-22.

21 F. Carriere, C. Withers-Martinez, H. van Tilberugh, A. Roussel, C. Cambillau and R. Verger, Biochim. Biophys. Acta, Rev. Biomembr., 1998, 1376, 417-432. 